\title{
De I'huile de foie de morue aux huiles de poisson concentrées, riches en acides gras polyinsaturés
}

\author{
Vue d'ensemble des techniques de production d'huile de poisson
}

Anne-Gaëlle GROSDEMANGE

POLARIS SA,

Moulin du Pont,

29170 Pleuven,

France

<ag.grosdemange@polaris.fr>

\begin{abstract}
Traditionally fish oil industry was associated to fish meal industry and the processing methods were mostly adapted to the raw material form: whole fish or part of fish (liver, head, by-products). Now, the processing line are more and more technically advanced when the customer requests for deodorized, uncontaminated and rich in PUFA oils increase. New process of extraction as cold press extraction emerges coming from vegetable oil industry. Refining including Deodorization and Decontamination is completed by stripping and molecular distillation decreasing the level of contaminants close to the detection limit of analytical methods. The lipochemistry allows to concentrate the natural content of omega 3: esterification then concentration by molecular distillation or $\mathrm{CO}_{2}$ supercritical plus transesterification (chemical/enzymatic) for TG form. Possibility of fish oil process is "unlimited" to offer to the market the benefice of omega 3 with safe, traceable, tasty fish oils.
\end{abstract}

Key words: fish oil, omega 3/PUFA, extraction, concentration, refining, esterification, lipochemistry

premières de qualité, approvisionnement qui se doit de garantir une traçabilité et la préservation des espèces. Historiquement, les process de production de ces huiles étaient essentiellement adaptés à la forme de la matière première, c'est-à-dire des poissons entiers ou des parties telles que la tête, le foie ou les sous-produits et ne prenaient que peu en compte la «qualité finale de l'huile ». Aujour$\mathrm{d}^{\prime}$ hui la technologie de pointe s'adapte aux demandes, aux exigences réglementaires et aux attentes des consommateurs en élaborant des huiles désodorisées, décontaminées ou concentrées en acides gras polyinsaturés.

\section{Méthodes d'extraction d'huile de poisson}

L'extraction historique de I'huile de foie de morue à partir de foies frais (ou congelés) se réalise par la cuisson des foies puis par un broyage et une centrifugation pour séparer I'huile des protéines et de l'eau.

L'extraction la plus courante d'huile de poisson est celle pratiquée sur les poissons bleus de type harengs, sardines, maquereaux, anchois, dans les usines mixtes de farines et d'huiles de poissons (figure 1). Ce process enchaîne des étapes de cuisson et pressage de poissons entiers pour séparer d'une part les protéines récupérées dans la fraction farine et les lipides d'autre part récupérés dans l'huile.
Ce type d'extraction par pression à chaud a pour principal inconvénient la cuisson de l'huile, entraînant une dégradation de la qualité oxydative de l'huile et une perte de teneurs en EPA et DHA. D'autres procédés innovants arrivent sur le marché tels que l'extraction par pression à froid, process breveté issu de l'industrie de production des huiles végétales et en particulier de l'extraction d'huile d'olive.

Il existe d'autres méthodes d'extraction moins courantes telles que l'extraction par voie enzymatique. Cette technique est basée sur l'action de protéases spécifiques à basse température $\left(60^{\circ} \mathrm{C}\right)$ sur les tissus protéiques.

\section{Méthodes de raffinage}

Après extraction, I'huile obtenue est une huile brute, contenant des lipides saturés ou non, polaires ou apolaires, des composés insaponifiables et de possibles traces d'eau ou de contaminants lipophiles de l'environnement selon l'origine et les espèces de poissons.

Afin d'éliminer les composés indésirables, comme par exemple les lipides saturés ou les contaminants, I'huile doit subir un raffinage. Le raffinage est une succession d'étapes de traitements physiques et chimiques en ordre et nombre variables en fonction du degré de raffinage de I'huile que I'on souhaite obtenir (figure 2). 


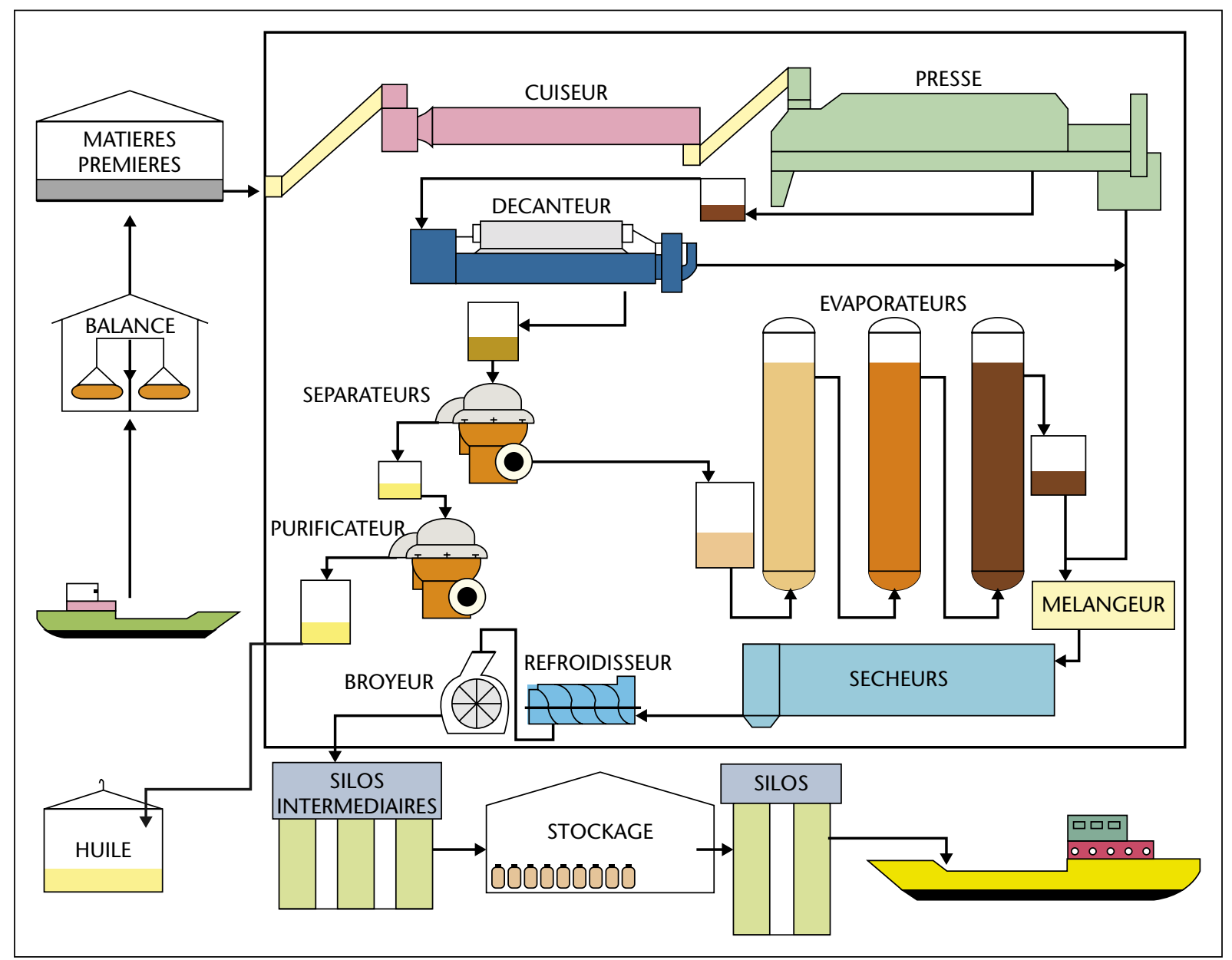

Figure 1. Obtention d'huile et de farine de poissons (Source IFFO).

La première étape est l'étape de démucilagination (ou dégommage) qui consiste à ajouter de l'acide phosphorique et de l'eau. Cette étape permet d'éliminer les composés polaires tels que les phospholipides, les lécithines, les pigments et également certains contaminants comme les métaux lourds.

L'étape suivante est la neutralisation. Elle se réalise par l'ajout de soude afin d'éliminer les acides gras libres sous forme de savon.

L'étape de cristallisation (winterisation) est classique pour certaines huiles à forte teneur en acides gras saturés telles que les huiles de thon par exemple. Elle permet, par refroidissement de l'huile à $0-4{ }^{\circ} \mathrm{C}$, de figer les acides gras saturés, tels que la stéarine, qui restent ensuite piégés dans un filtre-presse. Par exemple, I'huile de thon, qui sans étape de winterisation est naturellement trouble, est ainsi clarifiée. La décoloration ou le blanchiment élimine les colorants et autres substances indésirables par filtration de l'huile sur des terres de type charbon activé ou de silice amorphe. L'étape de décoloration est une étape majeure de décontamination, le choix des terres de filtration pouvant optimiser et cibler le captage de certains contaminants. Les sites de raffinage ont de plus en plus recours à une étape de décontamination complémentaire par distillation, à l'exemple de la méthode brevetée short path stripping distillation qui amène le niveau de contamination à des seuils proches des limites de détection analytiques, et ce, indépendamment de la contamination initiale de l'huile brute.

La dernière étape de raffinage élimine les produits odorants et volatils par injection de vapeur d'eau. L'opération est réalisée sous vide, les composés volatils sont entraînés lors du soutirage. Les progrès réalisés ces dernières années dans la technique de désodorisation et la préservation des qualités oxydatives initiales de I'huile, par l'arrivée de méthode d'extraction douce, permettent d'obtenir des huiles de poisson ultradésodorisées que le consommateur néophyte ne pourra pas différencier à l'odeur ni au goût d'une huile végétale.
L'huile en sortie de raffinage est stabilisée par I'ajout d'antioxydants tels que des tocophérols, puis conditionnée sous atmosphère inerte (azote ou argon).

\section{Méthodes de concentration en oméga-3}

Il est possible, avec les nouvelles techniques, de concentrer les huiles naturellement riches en oméga-3.

Les teneurs naturelles des huiles de poisson sont de l'ordre de $8 \%$ d'EPA et de $9 \%$ de DHA pour les huiles de foie de morue ou les huiles de saumon et de 20 à $30 \%$ d'EPA et de DHA pour les huiles de poissons gras. Les standards du marché pour ces dernières sont $5 \%$ d'EPA et $25 \%$ de DHA pour les huiles de thon et $18 \%$ d'EPA et $12 \%$ de DHA pour les huiles de poissons bleus (ratio obtenu par des mélanges/assemblages de lots). 


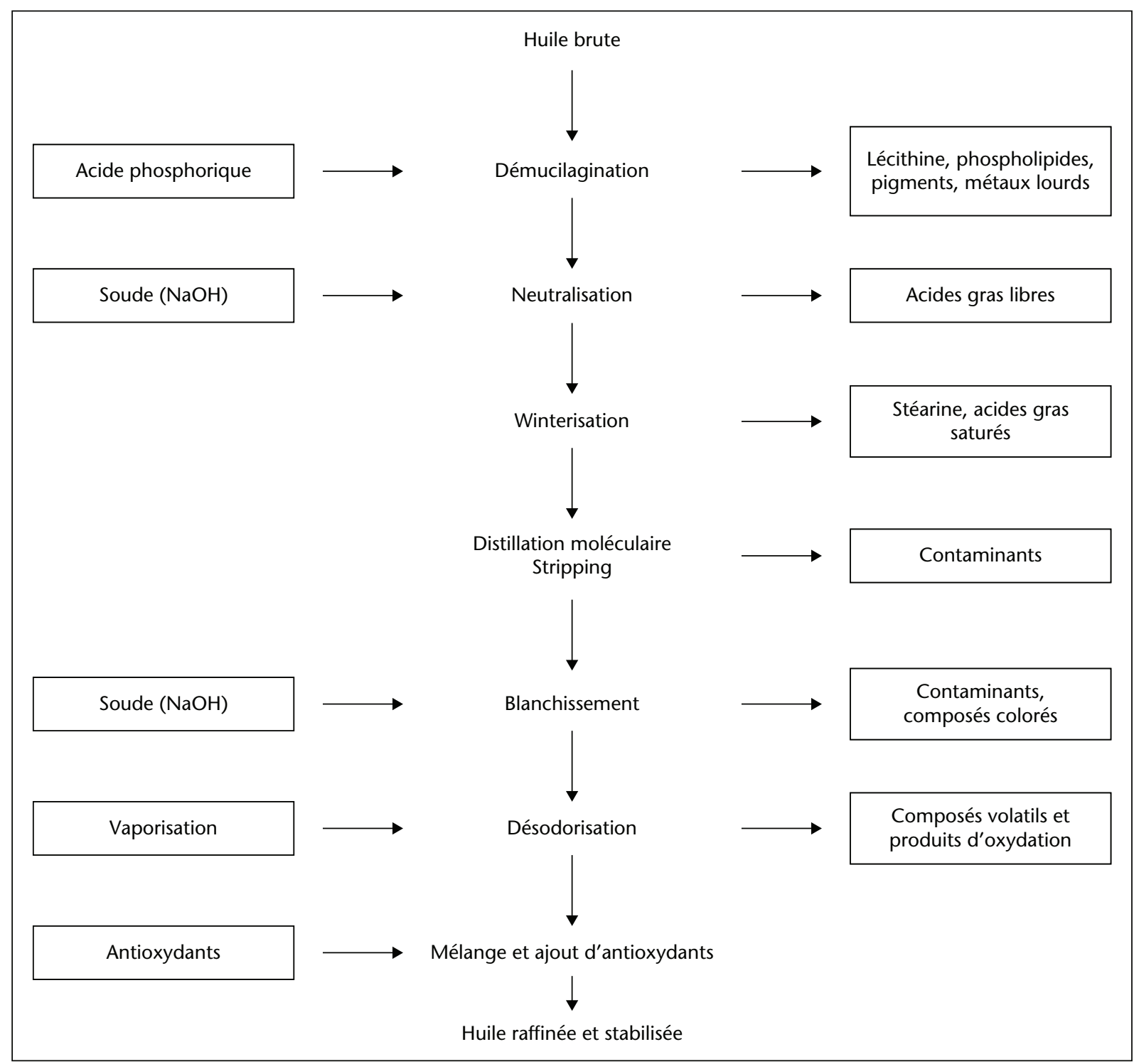

Figure 2. Procédé classique de raffinage.

Les huiles de poisson concentrées proposent des teneurs environ doubles des teneurs précédentes soit $50 \%$ de DHA pour une huile de thon concentrée et peuvent aller jusqu'à des teneurs de $90 \%$ en DHA ou en EPA.

\section{Concentration par winterisation}

La concentration par winterisation permet un gain de quelques points en EPA et en DHA. En effet, cette étape effectuée lors du raffinage (voir description plus haut) élimine les acides gras saturés cristallisés à basse température, ce qui a pour conséquence une légère concentration, proportionnellement en acides gras mono- et polyinsaturés. Cependant, cette concentration n'est que faible et relative, car une partie des acides gras insaturés, dont des oméga-3, est également entraînée avec la fraction stéarique.

\section{Concentration par voie chimique ou par voie enzymatique}

La concentration par voie chimique par ajout de sodium éthylate ou par voie enzymatique par ajout de lipase comporte deux étapes (figure 3) : l'éthanolyse spécifique des acides gras sous forme initiale de triglycérides par ajout d'éthanol générant du glycérol, d'une part, et des acides gras oméga-3 sous forme d'éthyl-esters volatils, d'autre part. La fraction en oméga-3 est ensuite concentrée par distillation en un ou deux passages des ces éthylesters volatils.

\section{Extraction et/ou concentration par fluide supercritique}

Les procédés d'extraction d'AGPI utilisant des solvants organiques permettent d'obtenir des rendements élevés mais nécessitent ensuite I'élimination des résidus de solvant. L'utilisation de fluide supercritique, $\mathrm{CO}_{2}$ par exemple, est un procédé alternatif en raison de ses propriétés non inflammable, compatible avec l'environnement et sa nature inerte. Dans ce cas, I'huile est au préalable estérifiée et subit un fractionnement à l'urée, entraînant les acides gras saturés ainsi cristallisés. Le principe de la concentration repose sur le $\mathrm{CO}_{2}$ qui, à l'état supercritique, se comporte comme un fluide d'extraction et entraîne les acides gras. En plusieurs passages, leurs concentrations 


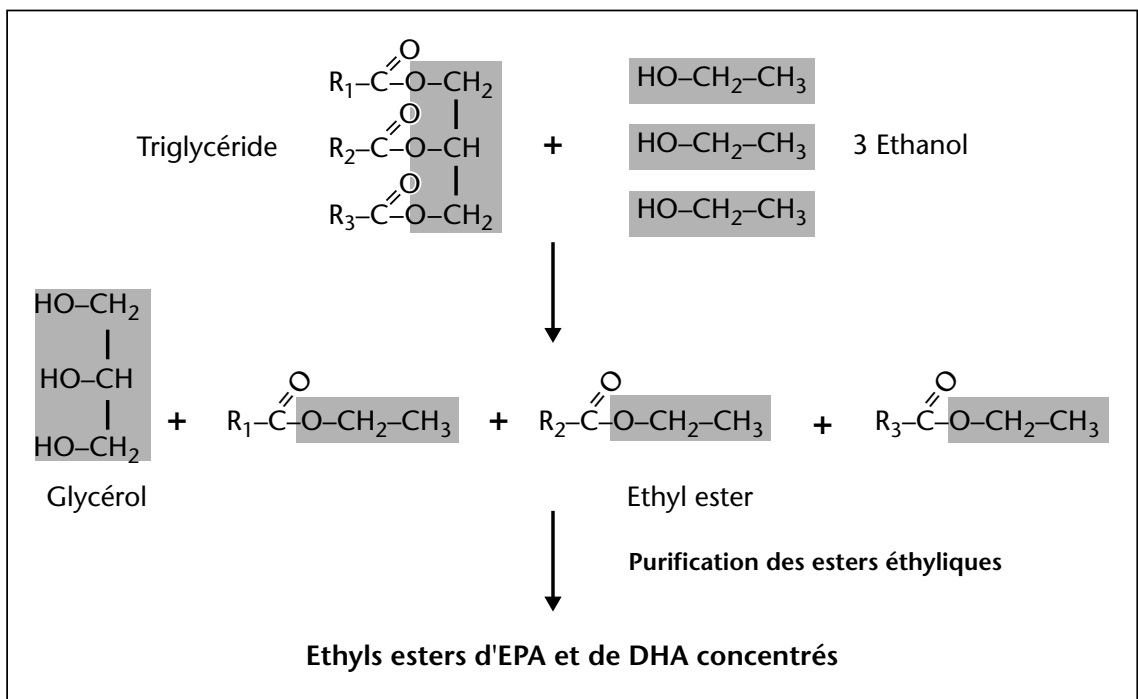

Figure 3. Procédé de concentration par éthanolyse et purification.

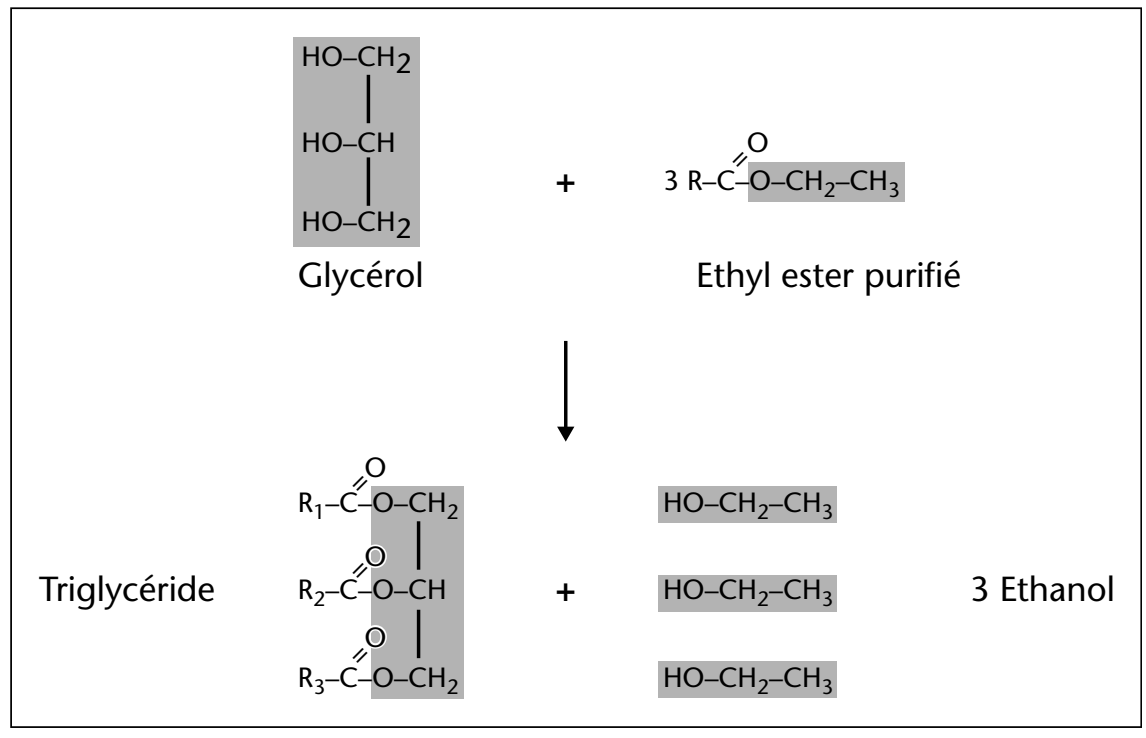

Figure 4. Obtention de triglycérides concentrés par transestérification enzymatique.

augmentent donc. Cette méthode permet $d^{\prime}$ atteindre une concentration très élevé à faible coût (le $\mathrm{CO}_{2}$ étant recyclable) mais nécessite des installations spécifiques et la séparation fluide/acides gras est complexe.

\section{Formes d'acides gras oméga-3}

Les huiles concentrées issues des étapes de concentration précédemment décrites sont glycérol, elle aboutit à la production de triglycérides concentrés présentant la même teneur en EPA et/ou DHA et à l'élimination de l'éthanol. Ce process enzymatique a pour avantages une large sélectivité, des conditions de manipulation douces, la possibilité d'une réutilisation de l'enzyme si elle est immobilisée, une grande activité et stabilité, donne à I'huile une meilleure stabilité vis-à-vis de l'oxydation ainsi qu'une meilleure odeur et un meilleur goût. Enfin, la concentration enzymatique produit moins de composés indésirables.

Les progrès réalisés en lipochimie permettent également de réaliser des greffages par voie chimique et/ou enzymatique pour proposer des formes d'oméga-3 innovantes: monoglycérides, diglycérides, phospholipides qui ouvrent de nouvelles opportunités de par leurs hautes biodisponibilités.

\section{Conclusion}

Nous avons passé en revue les principales techniques d'extraction et de concentration des huiles de poisson. Les possibilités de process pour obtenir des huiles de poisson semblent illimitées au vu des progrès de la lipochimie. Demain, le challenge de la filière sera d'offrir des huiles de qualité irréprochable tant sur le plan organoleptique, avec des huiles ultradésodorisées, que sur le plan de la sécurité alimentaire, avec des attentes de "zéro " contaminant. Les teneurs en oméga-3 souhaitées seront toujours de plus en plus élevées à des coûts raisonnables. En parallèle, on note une évolution vers des process de plus en plus « propres » type chimie verte et chimie bleue. Afin d'offrir aux consommateurs des huiles riches en oméga-3, dont les bénéfices sur la santé sont largement démontrés le challenge portera aussi sur des formes hautement biodisponibles pour des applications santé ciblées.

\section{RÉFÉRENCES}

Riediger ND, Othman RA, Suh M, Moghadasian MH. A systemic review of the roles of $\mathrm{n}-3$ fatty acids in health and disease. / Am Diet Assoc 2009; 109: 668-79.

AFSSA. Acides gras de la famille oméga 3 et système cardiovasculaire : intérêt nutritionnel et allégations. Juillet 2003. www.afssa.fr.

IFFO: International Fishmeal and Fish oil Organization. www.iffo.net. 